\title{
Utilisation de la sélection génomique chez les bovins laitiers
}

\author{
F. GUILLAUME ${ }^{1,2,3}$, D. BOICHARD ${ }^{1,2}$, V. DUCROCQ ${ }^{1,2}$, S. FRITZ ${ }^{4}$ \\ ${ }^{1}$ INRA, UMR1313 Génétique Animale et Biologie Intégrative, F-78352 Jouy-en-Josas, France \\ 2 AgroParisTech, Génétique Animale et Biologie Intégrative, 16 rue Claude Bernard, F-75231 Paris, France \\ 3 Institut de l'élevage, 149 Rue de Bercy, F-75595 Paris, France \\ 4 UNCEIA, 149 Rue de Bercy, F-75595 Paris, France \\ Courriel :Francois.guillaume@jouy.inra.fr
}

Les schémas de sélection bovins laitiers ont été parmi les premiers à tirer parti des outils moléculaires afin d'améliorer leur efficacité. Depuis dix ans, ces outils ont été intégrés aux schémas de sélection français, néanmoins la rupture technologique que constituent les puces denses de SNP disponibles depuis 2008, aura marqué des changements profonds à la fois des schémas et de la sélection en général tant au niveau français qu'international.

L'espèce bovine cumule un certain nombre de freins au progrès génétique : gestation longue (9 mois), maturité sexuelle tardive (15 mois), espèce monotoque, nombreux caractères d'intérêt agronomique dont l'expression, souvent tardive, est liée au sexe. Pour pallier ces limitations, les schémas de sélection bovins ont intégré successivement de nombreuses biotechnologies (insémination artificielle, transplantation embryonnaire) et méthodes de sélection (testage sur descendance, évaluation BLUP) afin d'être les plus efficaces possible. La plus récente évolution en date est l'usage d'informations moléculaires pour l'évaluation génétique. Nous présenterons dans cet article l'impact et la mise en œuvre pratique de cette innovation sur les schémas de sélection.

\section{1 / Les travaux pionniers}

L'essor des outils moléculaires a ouvert de nouvelles perspectives pour la compréhension du vivant et par voie de conséquence sa gestion. Durant les années 90, de nombreux travaux postulant l'utilisation d'informations moléculaires afin d'améliorer la précision des évaluations génétiques des candidats à la reproduction ont conclu à l'impact positif de telles approches sur le progrès génétique (Kashi et al 1990). L'intérêt de cette intégration pouvait néanmoins être réduit dans le cas de caractère à héritabilité forte (Lande et Thompson 1990), ou si le nombre de Quantitative Trait Loci (QTL) ou plutôt la proportion de variance génétique expliquée par les QTL était faible (Meuwissen et
Goddard 1996, Spelman et al 1999). Les imprécisions sur la localisation des QTL ainsi que sur l'estimation de la proportion de la variance génétique expliquée par ceux-ci pouvaient quant à elles réduire de moitié le progrès génétique supplémentaire permis par l'information QTL (Spelman et Bovenhuis 1998). Ainsi, il est apparu très tôt que si l'intérêt de ces outils moléculaires pour la sélection semblait évident, leur utilisation efficace demeurait contrainte par des facteurs difficiles à maîtriser.

\section{1 / Le premier programme français de sélection assistée par marqueurs}

A la suite des travaux pionniers de Georges et al (1995), de nombreux travaux de détection de QTL ont été mis en œuvre à travers le monde (Khatkar et al 2004 pour une revue). En France, un programme de détection de QTL conduit de 96 à 99 (Boichard et al 2003), a abouti à la mise en évidence de plusieurs centaines de QTL. La taille du dispositif restant réduite, la localisation des QTL et les parts de variances qu'ils expliquaient portaient à caution et nécessitaient confirmation. Néanmoins, puisqu'un ensemble de QTL semblait faire consensus et que ces derniers permettaient d'expliquer des proportions de variance suffisamment importantes, il devenait envisageable de mettre en œuvre une évaluation assistée par marqueurs.

Un premier programme de sélection assistée par marqueurs a donc été mis en place en France dans les trois principales races bovines laitières
(Prim'Holstein, Normande et Montbéliarde). Il devait permettre d'une part de fournir aux professionnels un outil d'aide à la sélection mais également de collecter des informations afin de progresser dans la connaissance du vivant (Fritz et al 2003).

Une évaluation assistée par marqueurs de première génération (SAM1) a été réalisée mensuellement de 2001 à 2008. Cette évaluation reposait sur un modèle mixte de type Fernando et Grossman (1989), postulant un déséquilibre de liaison familiale (Robert-Granié et al 2011). Dans le détail, 43 marqueurs microsatellites ont été utilisés pour suivre 14 régions QTL impliquées dans le déterminisme génétique de 7 caractères évalués. Ces caractéristiques, paraissant modestes aujourd'hui, correspondaient à un compromis acceptable entre coûts de génotypage et gains de précision des évaluations au vu des connaissances de l'époque.

Les évaluations de la SAM1 ont avant tout été utilisées pour sélectionner les jeunes mâles les plus prometteurs à tester sur descendance notamment au sein de fratries, ce qui présentait un grand intérêt puisqu'une proportion importante $-95 \%$ d'après Colleau et al (1998) des taureaux testés sur descendance sont issus de transplantation embryonnaire. Cette utilisation a impliqué un travail important d'organisation afin de pouvoir permettre de prélever, génotyper et évaluer un candidat en moins de 3 mois après sa naissance, ce qui a nécessairement engendré un coût supplémentaire au niveau des schémas. Ces surcoûts pouvaient être facilement 
compensés par une réduction du nombre d'animaux testés, mais également par une évolution des prix versés aux éleveurs propriétaires des veaux, réduisant les revenus de ces derniers (Boichard et al 2006). Les évaluations ont également dans une moindre mesure permis une meilleure gestion des femelles des noyaux de sélection notamment dans le choix de pleines sœurs issues d'une transplantation embryonnaire. Les évaluations étaient alors utilisées afin de ne transplanter que la meilleure des sœurs, ceci permettant d'une part de réduire les coûts de schéma mais également de contenir l'accroissement de la consanguinité. Par construction, les évaluations de la SAM1 ne pouvaient pas avoir une portée générale. Néanmoins elles étaient pertinentes dans les cas, fréquents, de larges fratries d'animaux, qui auparavant auraient été exploités trop intensivement dans le schéma.

Les gains de précision permis par ces évaluations demeuraient modérés (Guillaume et al 2008a et b), mais en accord avec les potentialités du dispositif. De plus, l'accumulation d'informations et la collecte ciblée d'informations sur des filles de testage par exemple permettaient d'améliorer parfois substantiellement la précision des évaluations. Au final, le programme de sélection assistée par marqueurs mené de 2001 à 2008 aura permis la collecte de 70000 génotypages (Fritz et al 2007). Il aura été le support de nombreuses détection de QTL (Gautier et al 2006, Guillaume et al 2007). Il aura surtout préparé les entreprises de sélection françaises à intégrer l'évaluation assistée par marqueurs dans leurs schémas. Le lien fort tissé avec les entreprises a été également le support de discussions et de mise en place d'outils de gestion des populations dépassant le simple cadre de l'évaluation (Colleau et al 2006).

\section{2 / Initiatives internationales}

$\mathrm{Au}$ niveau international, d'autres initiatives équivalentes ont été mises en place, mais peu d'informations ont été rendu publiques. L'Allemagne a mis en place une évaluation assistée par marqueurs (Wilkens et al 2006). Les principales caractéristiques de ce programme étaient un génotypage à un âge assez tardif des mâles (limitant les économies sur l'achat de mâles), la ré-utilisation des marqueurs de contrôle de filiation ou de contrôles de maladie génétiques (qui a priori n'étaient pas les plus indiqués pour suivre les QTL) et l'utilisation des évaluations surtout ciblée sur le choix des femelles. Ces choix stratégiques ont sans doute diminué l'intérêt de l'outil. En Nouvelle-Zélande, un programme de sélection assisté par mar- queurs intra-famille a été mis en place dès 98 . Il devait permettre au sein d'un groupe de pleins-frères de ne retenir qu'un mâle à tester sur descendance. Du fait des modestes résultats de reproduction et faute de permettre une pression de sélection suffisante, ce programme a finalement été abandonné en 2000 (Spelman et al 2010).

Les initiatives connues aux EtatsUnis, semblent s'être heurtées à des difficultés de mise en place d'un réseau efficace de génotypages. Les Pays-Bas ont quant à eux mis en place un programme intégrant des mutations causales, peu d'informations ont été dévoilées sur ce programme, mais il semble que ce dernier ait régulièrement évolué pour s'adapter aux nouvelles technologies disponibles rendant ainsi difficile la constitution d'une base de données large contenant des données homogènes.

De ce panorama rapide, il découle qu'au-delà du modèle d'évaluation, les facteurs clés de succès de ces premiers programmes d'évaluation assistée par marqueurs résidaient surtout dans l'implémentation de ces programmes sur le terrain.

\section{2 / Le génotypage haut débit et la sélection géno- mique}

A partir de 2007, le génotypage à haut débit (à l'aide de puces pangénomiques de SNP, telles que la BovineSNP50, Matukumalli et al 2009) et l'utilisation de nouveaux modèles d'évaluation ont permis d'atteindre des niveaux de précisions des index nettement supérieurs aux méthodes classiques (pour une présentation plus complète voir RobertGranié et al 2011) ouvrant une nouvelle ère pour la sélection.

Contrairement à la première génération d'évaluations assistées par marqueurs pratiquées par quelques schémas de sélection à travers le monde, cette seconde génération sera rapidement adoptée dans une majorité de pays et utilisée aussi bien dans les schémas de sélection que par les éleveurs. Nous présenterons par la suite quelques évolutions majeures engendrées par la sélection génomique.

\section{1 / Evolutions dans les schémas de sélection français}

En France, à partir d'octobre 2008 une évaluation intégrant l'effet de QTL suivis à l'aide d'haplotypes de $6 \mathrm{SNP}$ a été mise en œuvre mensuellement pour 15 caractères, dans les trois principales races laitières (Prim'Holstein, Normande et Montbéliarde). Du fait de l'expérience acquise depuis 2001 cette évolution a été intégrée par les entreprises de sélection avec une rapidité et une simplicité remarquables (Fritz et al 2010). Dès lors, les bases de données se sont enrichies régulièrement selon un rythme d'environ un millier de typages additionnels par mois. Dès juin 2009, le nombre de caractères évalués s'est accru (atteignant 25 à 28 caractères évalués selon les races) les paramètres génétiques des modèles se sont affinés et l'évaluation a été officiellement reconnue. Contrairement aux évaluations de la SAM1, les schémas de sélection disposaient désormais d'un indicateur de la valeur génétique des jeunes candidats exhaustif (la plupart des caractères étant indexés), plus fiable et permettant des comparaisons entre individus non apparentés. Ces caractéristiques ont logiquement conduit à augmenter l'intensité de sélection des jeunes candidats mâles.

$\mathrm{Au}$ niveau de l'approvisionnement des schémas de sélection, les jeunes taureaux évalués seulement sur la base de leur évaluation génomique ont été utilisés à raison de 20 à $50 \%$ des inséminations de pères à taureaux dès 2010 . Ainsi, dès juin 2011, leurs premiers descendants sont proposés par les entreprises de sélection. Ce type de candidats, ayant un intervalle de génération de 2 à 3 ans avec leur père - contre 6 ans classiquement -, devrait constituer une part de plus en plus importante de la population de mâles diffusés, pour vraisemblablement devenir la norme d'ici quelques années. Ce changement dans les schémas de sélection permet de rapprocher l'intervalle de génération de sa valeur optimale et d'améliorer grandement le progrès génétique.

De plus, les jeunes pères à taureaux utilisés dans les schémas sont plus nombreux et chacun moins diffusés que leurs alter ego testés sur descendance, ceci afin de compenser la plus faible fiabilité de leurs index, mais également du fait de la relative abondance de taureaux aux profils similaires, tant et si bien qu'aucun taureau ne semble justifier d'être plus utilisé qu'un autre. Cette organisation de schéma tend à suivre les recommandations de Colleau et al (2009) qui garantissent un gain de progrès génétique important tout en contenant l'augmentation de la consanguinité.

Les mères à taureaux connaissent une situation inédite. Elles peuvent désormais être évaluées avec une fiabilité (Coefficient de Détermination, CD) identique à celui des taureaux pour l'ensemble des caractères évalués en routine, notamment pour les caractères fonc- 
tionnels dont l'importance économique est prépondérante pour les éleveurs. L'augmentation de la fiabilité des évaluations génétiques des femelles d'au minimum $20 \%$ impactera favorablement le progrès génétique. De plus, la sélection peut être plus équilibrée car ne favorisant pas, par construction, les seules femelles fortes en production. La population de reproducteurs d'élite se trouve potentiellement nettement élargie et devrait pouvoir permettre de mettre en avant de nouveaux reproducteurs aux caractéristiques originales et désirables. Ce phénomène devrait être encore amplifié dans les années à venir avec la massification du génotypage des femelles laitières qui devrait permettre l'évaluation génomique de plusieurs dizaines de milliers de femelles chaque année. Enfin, le recours à de multiples transplantations embryonnaires sur les femelles à la fois originales et à haut potentiel génétique devrait permettre d'impacter le progrès génétique tout en maintenant la variabilité génétique du noyau de sélection.

La sélection génomique s'est donc traduite dans les schémas de sélection par une utilisation de reproducteurs à la fois plus jeunes, plus nombreux et plus originaux. Contrairement au premier programme de sélection assistée par marqueurs, les évaluations génomiques interviennent fortement sur chacune des voies de la sélection et permettent d'envisager de réels gains de progrès génétique, là où auparavant un simple retour sur investissement était jusqu'alors espéré. Avec des intervalles de générations quasiment réduits à leur minimum, une fiabilité élevée des évaluations pour une population soumise à la sélection élargie, il reste désormais peu de facteurs du progrès génétique pouvant connaître de grande optimisation.

\section{2 / Evolution dans les élevages}

Le dispositif d'amélioration génétique s'est basé durant des années sur le testage sur descendance. Or, ce dispositif très onéreux a vu son utilité réduite avec l'arrivée de la génomique. Désormais, les jeunes mâles des schémas de sélection ont des profils globalement bien connus (contrairement aux anciens taureaux de testage). Ils peuvent donc à l'instar des taureaux de service être utilisés de manière ciblée pour réaliser des accouplements raisonnés. De plus les performances des premières filles de ces taureaux n'apporteront potentiellement qu'un gain marginal de CD. En conséquence, les jeunes taureaux sont désormais «diffusés» et non plus testés. En pratique, les éleveurs achètent les doses de ces taureaux à un prix plus modéré en fonction de leurs objectifs de sélection et ne sont plus rétribués pour conserver et faire produire les femelles issues de ces jeunes taureaux.

La diffusion des jeunes reproducteurs se fait souvent par le biais de gammes de taureaux aux profils équivalents. Ce choix permet de garantir aux entreprises de sélection un nombre important et homogène de descendantes pour chacun des taureaux diffusés, tout en limitant à l'échelle des élevages le risque de déceptions suite à l'usage trop intensif d'un nombre réduit de taureaux finalement décevants. Parallèlement, certains taureaux choisis pour leur attractivité commerciale sont également diffusés individuellement. Cette situation est néanmoins plus risquée car elle maintient un «star système», responsable de dérives par le passé.

L'utilisation de jeunes taureaux a été globalement très vite intégrée par les professionnels français. Ainsi dès la campagne 2009-2010, selon les races de 15 à $20 \%$ des inséminations ont été réalisés avec des taureaux sélectionnés sur la base de leur évaluation génomique, la répartition du nombre d'inséminations par taureau restant généralement contenue de 1000 à 2000 doses (à comparer aux dizaines de milliers d'inséminations réalisées par les taureaux testés sur descendance les plus diffusés). Dans les faits, le risque d'une utilisation excessive d'un jeune taureau est réduit d'une part par la production plus limitée des jeunes taureaux (et donc d'une pénurie possible de ses doses) et d'autre part par la relative abondance en bons jeunes taureaux.

La diffusion large et homogène des jeunes taureaux, ne peut se faire qu'au prix d'un changement de paradigme pour les éleveurs (Colleau et al 2009). On passe ainsi d'un système très «individu centré», où les mâles reproducteurs sont rares et coûteux, à un système de relative abondance où les mâles reproducteurs sont plus nombreux, interchangeables et remplaçables : sitôt leur stock de doses produit, la plupart d'entre eux seront reformés. Or, ce paradigme est à la base de l'intérêt et la passion qu'ont certains éleveurs pour la sélection. Les entreprises de sélection devront donc veiller à maintenir la participation et l'implication de leurs éleveurs au schéma de sélection s'ils veulent toujours pouvoir disposer d'un pool important et varié de reproducteurs.

Des premiers travaux d'évaluations internationales de jeunes taureaux non évalués sur descendance ont débuté et devraient permettre la diffusion internationale des jeunes reproducteurs les plus prometteurs. Néanmoins, du fait de la gestion de la production de semences des jeunes reproducteurs, il est désormais possible que des entreprises de sélection fassent une exploitation exclusive de taureaux à grand potentiel génétique. Ce cas de figure, fréquent dans d'autres filières, pourrait présenter des avantages mais également un risque en termes de variabilité génétique.

\section{3 / Evolution internationale}

Le développement de la sélection génomique est un phénomène assez global, mais paradoxalement il existe encore actuellement une grande variabilité dans l'application de cette technologie. Ainsi, aucune méthodologie d'évaluation génomique ne s'est réellement imposée jusqu'ici (Robert-Granié et al 2011). Il en va de même en termes de mise en œuvre pratique de la sélection génomique. La seule véritable tendance internationale amorcée est la constitution de grands consortia visant à échanger des génotypes entre pays afin de constituer des populations de référence suffisantes.

En Holstein, depuis 2009, le consortium Eurogenomics, regroupant la France, l'Allemagne, les Pays-Bas et les pays scandinaves a permis la mise en commun des génotypes pour 50000 SNP de 16000 taureaux Holstein testés sur descendance. Dès 2008, les EtatsUnis et le Canada ont également échangé leurs génotypes pour améliorer les évaluations. Depuis lors, d'autres pays les ont rejoints. Ceci indique clairement l'importance capitale de constituer des populations de références importantes, mais également la prise de conscience de l'intérêt de collaborer internationalement dans l'intérêt commun.

A l'instar du projet Eurogenomics, une collaboration internationale équivalente, le projet Intergenomics a été mise en place en race brune sous l'égide d'Interbull (centre d'évaluations internationales). Cette initiative est actuellement l'une des seules possibilités pour les races à plus faible effectif de pouvoir accéder à la sélection génomique.

$\mathrm{Au}$ niveau international, différentes stratégies co-existent et révèlent la grande variété de structuration des filières de sélection mais aussi d'appropriation des nouveaux outils de la génomique. Ainsi, aux Etats-Unis, certaines entreprises maintiennent ou même développent un testage sur descendance de taureaux présélectionnés sur leur information génomique en contractualisant des élevages et en diffusant des jeunes taureaux sous formes de packs, tandis que d'autres entreprises misent plus sur la commercialisation de jeunes taureaux ciblés avec éventuellement le recours au sexage. 
Au sein des schémas de sélection, l'intégration de jeunes pères à taureaux non testés sur descendance s'impose peu à peu. Selon une enquête biannuelle sur 28 entreprises de sélection internationales en octobre 2008, une seule annonçait utiliser des jeunes pères à taureaux dans son schéma, un an plus tard ce chiffre était de 7 et enfin en 2010, 13 entreprises de sélection déclaraient utiliser des jeunes pères à taureaux. Cette utilisation représente en moyenne $40 \%$ des accouplements du schéma de sélection avec des valeurs allant de 99 à $5 \%$. Les entreprises de sélection n'utilisant toujours pas de jeunes taureaux dans leurs schémas sont en fait celles dont le pays ne dispose pas d'évaluations génomiques officielles. Il existe pour elles un risque réel de marginalisation, d'autant plus que l'utilisation de jeunes reproducteurs internationaux n'est pas forcément aisée. La commercialisation de jeunes reproducteurs sur le marché international, n'en est qu'à ses débuts. Elle se base toujours sur un schéma traditionnel, mettant en avant un nombre très réduit de reproducteurs ayant souvent un stock de doses limité et pour lesquels seule une évaluation génomique sur l'échelle du pays d'origine est disponible. Ceci représente une réelle régression par rapport à la situation actuelle pour les taureaux testés sur descendance. Dans ces conditions, l'intérêt de jeunes taureaux étrangers non testés sur descendance peut sembler discutable au sein des schémas de sélection. La validation des méthodes d'évaluations génomiques par Interbull ainsi que la mise en place d'une évaluation internationale génomique (S-GMACE) pourraient améliorer la situation en facilitant la comparaison entre taureaux de pays différents.

Si les outils de la sélection génomique ont bien été intégrés par la plupart des grands pays d'élevage, il est encore trop tôt pour tirer des conclusions sur l'utilisation qui sera faite de ces derniers, de nombreux ajustements et changements stratégiques devraient avoir encore lieu dans les années à venir. Plus que la méthode d'évaluation génomique, ou la taille de la population de référence, ce sont les modalités d'utilisation de ces outils qui conditionneront la compétitivité des schémas.

\section{Conclusion}

Si les marqueurs moléculaires ne permettent que d'améliorer la précision des évaluations, ils sont un élément central qui a amené les schémas de sélection bovins laitiers à travers le monde à évoluer pour gagner en efficacité. L'intégration des évaluations génomiques par la filière bovine laitière française s'est faite relativement rapidement et tend à converger vers les pratiques recommandées pour augmenter le progrès génétique tout en ménageant la variabilité génétique. Au niveau international, les outils de la sélection génomique ont également été intégrés mais selon des modalités différentes de celle de la France. L'avenir nous permettra d'évaluer la pertinence de chacun des choix. Enfin, si la sélection génomique est bel et bien intégrée dans le paysage de l'élevage bovin laitier français, elle reste un objectif à atteindre pour certains pays mais également pour des races laitières à plus faibles effectifs. Permettre un accès à cette technologie dans les situations plus complexes reste ainsi un grand défi pour l'avenir.

\section{Références}

Boichard D., Grohs C., Bourgeois F., Cerqueira F., Faugeras R., Neau A., Rupp R., Amigues Y., Boscher M.Y., Levéziel H., 2003. Detection of genes influencing economic traits in three french dairy cattle breeds. Genet. Sel. Evol., 35, 77-101.

Boichard D., Fritz S., Rossignol M.N., Guillaume F., Colleau J.J., Druet T., 2006. Implementation of marker selection: practical lessons from dairy cattle. Proc. $8^{\text {th }}$ World Congr. Genet. Appl. Livest. Prod., 22-11.

Colleau J.J., Heyman Y., Renard J.P., 1998. Les biotechnologies de la reproduction chez les bovins et leurs applications réelles ou potentielles en sélection. INRA Prod. Anim., 11, 41-56.

Colleau J.J., Regaldo D., Moureaux S., Barbat A., Fritz S., de Preaumont H., Tual K., Mattalia S., 2006 Mise en place de la gestion optimisée de la variabilité génétique dans les populations sélectionnées de bovins laitiers. Renc. Rech. Rum., 235-238.

Colleau J.J., Fritz S., Guillaume F., Baur A., Dupassieux D., Boscher M.Y., Journaux L., Eggen A., Boichard D., 2009. Simulation des potentialités de la sélection génomique chez les bovins laitiers. Renc. Rech. Rum., 419.

Fernando R.L., Grossman M., 1989. Marker assisted selection using best linear unbiased prediction. Genet. Sel. Evol., 21, 467-477.

Fritz S., Colleau J.J., Druet T., Boscher M.Y., Rossignol M.N., Malafosse A., Boichard D., 2003. Mise en place d'une sélection assistée par marqueurs dans les trois principales races bovines laitières françaises. Renc. Rech. Rum., 53-56.
Fritz S., Druet T., Guillaume F, Boscher M.Y., Eggen A. Gautier M., Colleau J.J., Boichard D., 2007. Bilan du programme de Sélection Assistée par Marqueurs dans les trois principales races bovines laitières françaises et perspectives d'évolution. Renc. Rech. Rum., 129-132.

Fritz S., Guillaume F., Croiseau P., Baur A., Hoze C., Dassonneville R., Boscher M.Y., Journaux L., Boichard D., Ducrocq V., 2010. Mise en place de la sélection génomique dans les trois principales races françaises de bovins laitiers. Renc. Rech. Rum., 455-458.

Gautier M., Barcelona R.R., Fritz S., Grohs C., Druet T., Boichard D., Eggen A., Meuwissen T.H.E., 2006. Fine mapping and physical characterization of two linked quantitative trait loci affecting milk fat yield in dairy cattle on bta26. Genetics, 172 , 425-436.

Georges M., Nielsen D., MacKinnon M., Mishra A., Okimoto R., Pasquino A.T., Sargeant L.S., Sorensen A., Steele M.R., Zhao X., Womack J.E., Hoeschele I., 1995. Mapping quantitative trait loci controlling milk production in dairy cattle by exploiting progeny testing. Genetics, 139, 907-920.

Guillaume F., Gautier M., Jemaa S.B., Fritz S., Eggen A., Boichard D., Druet T., 2007. Refinement of two female fertility QTL using alternative phenotypes in French Holstein dairy cattle. Anim. Genet., 38, 72-74.

Guillaume F., Fritz S., Boichard D., Druet T., 2008a. Estimation by simulation of the efficiency of MAS in the French marker-assisted selection program in dairy cattle. Genet. Sel. Evol., 40, 91-102.
Guillaume F., Fritz S., Boichard D., Druet T., 2008b. Correlations of marker-assisted breeding values with progeny test breeding values for 899 French Holstein bulls. J. Dairy Sci., 91, 2520-2522.

Kashi Y., Hallerman E., Soller M., 1990. Marker-assisted selection of candidate bulls for progeny testing programmes. Anim. Prod., 51, 63-74.

Khatkar M.S., Thomson P.C., Tammen I., Raadsma H.W., 2004. Quantitative trait loci mapping in dairy cattle: review and metaanalysis. Genet. Sel. Evol., 36, 163-190.

Lande R., Thompson R., 1990. Efficiency of marker-assisted selection in the improvement of quantitative traits. Genetics, 124, 743-756.

Matukumalli L.K., Lawley C.T., Schnabel R.D., Taylor J.F., Allan M.F., Heaton M.P., O'Connell J., Moore S.S., Smith T.P., Sonstegard T.S., Van Tassel C.P., 2009. Development and characterization of a high density SNP genotyping assay for cattle. PLoS One. 2009;4(4):e5350

Meuwissen T.H.E., Goddard M.E., 1996. The use of marker haplotypes in animal breeding schemes. Genet. Sel. Evol., 28, 161176.

Robert-Granié C., Legarra A., Ducrocq V., 2011. Principes de base de la sélection génomique. In : Numéro spécial, Amélioration génétique. Mulsant P., Bodin L., Coudurier B., Deretz S., Le Roy P., Quillet E., Perez J.M. (Eds). INRA Prod. Anim., 24, 331-340.

Spelman R., Bovenhuis H., 1998. Genetic response from marker assisted selection in an outbred population for differing marker brac- 
ket sizes and with two identified quantitative trait loci. Genetics, 148, 1389-1396.

Spelman R.J., Garrick D.J., Van Arendonk J.A.M., 1999. Utilisation of genetic variation by marker assisted selection in commercial dairy cattle populations. Livest. Prod. Sci., 59, 51-60.
Spelman R.J., Arias J., Keehan M.D. Obolonkin V., Winkelman A.M., Johnson D.L., Harris B.L., 2010. Application of genomic selection in the New-Zealand dairy cattle industry. Proc the $9^{\text {th }}$ World Congr. Genet. AppL. Livest. Prod., 311.
Wilkens J., Bünger A., Reinhardt F., 2006. Marker Assisted Selection in german Holstein, method and applications. 10 ${ }^{\text {th }}$ QTL-MAS Workshop Salzburg.

\section{Résumé}

Au sein des espéces d'élevage, c'est sans nul doute dans le secteur des bovins latiers que l'expérience de l'usage des outils moléculaires à des fins de sélection est la plus longue. Bien qu'aujourd'hui la plupart des grands pays d'élevage laitier réalisent des évaluations génomiques, l'introduction de ces outils ne se fait pas de façon uniforme. Ceci peut s'expliquer par des conditions de mise en œuvre différentes entre pays. Nous dressons ici un panorama de l'utilisation qui est faite des outils de la sélection génomique en bovins laitiers au niveau international.

\section{Abstract}

Genomic selection in dairy cattle

Dairy cattle are undoubtedly the most experienced agricultural species regarding the integration of molecular information for selection purposes. Although most prominent countries run genomic evaluations, these tools are being integrated into selection schemes in various ways depending on the actual constraints of each country. We present a survey of the use of molecular information in dairy cattle throughout the world.

GUILLAUME F., BOICHARD D., DUCROCQ V., FRITZ S., 2011. Utilisation de la sélection génomique chez les bovins laitiers. In : Numéro spécial, Amélioration génétique. Mulsant P., Bodin L., Coudurier B., Deretz S., Le Roy P., Quillet E., Perez J.M. (Eds). INRA Prod. Anim., 24, 363-368. 
